\section{Etymologia: Buruli Ulcer}

\author{
Tony M. Korman, Paul D.R. Johnson, John Hayman
}

Author affiliations: Monash University, Melbourne, Victoria, Australia (T.M. Korman); Austin Health, Melbourne (P.D.R. Johnson); University of Melbourne, Melbourne (P.D.R. Johnson, J. Hayman)

DOI: https://doi.org/10.3201/eid2612.200744

To the Editor: The recent etymologia by Henry in the March 2020 issue of Emerging Infectious Diseases recounts the fascinating origin of the name Buruli ulcer (1). Further to the history, in 1948, pathologist Peter MacCallum first described the clinical features for 6 patients from Victoria, Australia, each with an ulcer with undermined edges on an arm or a leg, and the characteristic histopathologic findings, including extensive necrosis and abundant acid-fast bacilli without granuloma formation (2). Five of the patients were identified by general practitioners D.G. Alsop, L.E. Clay, and J.R. Searls from the city of Bairnsdale (thus, another eponym "Bairnsdale ulcer") (3). Glen Buckle and Jean Tolhurst at the Alfred Hospital in Melbourne established experimental animal infections, and eventually isolated the causative organism (2), which they later named Mycobacterium ulcerans (4). The growth of $M$. ulcerans required prolonged incubation at a temperature of $30^{\circ} \mathrm{C}-33^{\circ} \mathrm{C}(2)$, which was only realized after the inadvertent use of a faulty incubator.

In 1964, Clancey described a "new" mycobacterium causing chronic skin ulcers in Uganda that "resembled" M. ulcerans which he named "Mycobacterium buruli" (5). However, the causative organism of Buruli ulcer was subsequently recognized as Mycobacterium ulcerans, which had been originally described in Australia.

\section{About the Author}

Dr. Korman is an adjunct clinical professor at Monash University; Director, Monash Infectious Diseases; and Director of Microbiology, Monash Health, Clayton, Australia. He has a wide range of clinical, laboratory, and research interests.

\section{References}

1. Henry R. Etymologia: Buruli ulcer. Emerg Infect Dis. 2020;26:504. https://doi.org/10.3201/eid2603.ET2603

2. MacCallum P, Tolhurst JC, Buckle G, Sissons H. A new mycobacterial infection in man. J Pathol Bacteriol. 1948;60:93-122. https:// doi.org/10.1002/ path.1700600111

3. Johnson PD. Buruli ulcer in Australia. In: Pluschke G, Roltgen K, editors. Buruli ulcer. Mycobacterium ulcerans disease. New York: Springer; 2019. p. 61-76. https://doi. org/10.1007/978-3-030-11114-4
4. Fenner F. The significance of the incubation period in infectious diseases. Med J Aust. 1950;2:813-8. https:/ / doi.org/10.5694/j.1326-5377.1950.tb106945.x

5. Clancey JK. Mycobacterial skin ulcers in Uganda: description of a new mycobacterium (Mycobacterium buruli). J Pathol Bacteriol. 1964;88:175-87. https:/ / doi.org/10.1002/ path.1700880123

Address for correspondence: Tony M. Korman, Monash Infectious Diseases, Monash Health Centre, 246 Clayton Rd, Clayton, VIC 3168, Australia; email: tony.korman@monash.edu

\section{Arthritis Caused by MRSA CC398 in Patient without Animal Contact, Japan}

\author{
Anders R. Larsen, Jesper Larsen
}

Author affiliation: Statens Serum Institut, Copenhagen, Denmark

DOI: https://doi.org/10.3201/eid2612.202780

To the Editor: In their recent article, Nakaminami et al. describe a case of human infection caused by Panton-Valentine leucocidin (PVL)-positive livestock-associated methicillin-resistant Staphylococcus aureus clonal complex 398 (MRSA CC398) in Japan (1). S. aureus CC398 includes 2 major MRSA variants with distinct genetic and epidemiologic properties, a highly transmissible and virulent human variant comprising both PVL-positive and PVL-negative strains and a more benign PVL-negative livestockassociated variant (2). We have previously shown that, in Denmark, nearly all case-patients colonized or infected with PVL-positive MRSA CC398 strains of the human variant have links to countries in mainland Asia, where the strain is endemic in the community (3). Our analysis revealed the existence of 2 phylogenetically distinct lineages (L1 and L2) with unique sequence types (STs), ST398 linked to China and ST1232 linked to Vietnam, Thailand, and Cambodia. Besides being PVL-positive and belonging to ST1232, the isolate described by Nakaminami et al. (1) also shared other genetic and phenotypic characteristics with the L2 strains: it carried spa type t034 and SCCmec type $\mathrm{V}$ and was resistant to aminoglycosides (gentamicin), lincosamides (clindamycin), 